\title{
Pairs of Reactive Radicals as Probes of a Polymer Structure
}

\author{
Igor V Khudyakov ${ }^{1}$ and Peter P Levin ${ }^{2,3 *}$ \\ ${ }^{1}$ Department of Chemistry, Columbia University, New York, USA \\ ${ }^{2}$ Emanuel Institute of Biochemical Physics, Russian Academy of Sciences, Moscow, Russia \\ ${ }^{3}$ Semenov Institute of Chemical Physics, Russian Academy of Sciences, Moscow, Russia
}

*Corresponding author: Peter P Levin, Emanuel Institute of Biochemical Physics, Semenov Institute of Chemical Physics, Russian Academy of Sciences, Moscow, Russia.

Received Date: October 29, 2019

Published Date: November 06, 2019

\section{Introduction}

Polymer chemists used to study and evaluate macroscopic properties of polymers and polymer articles (hardness, adhesion, elongation, stiffness, Tg, abrasion-resistance, different thermal and mechanical properties, conductivity, etc.) Study of such properties and evaluation of polymers not necessarily requires understanding of their molecular structure. However, it is very desirable to understand polymer structure and properties at a molecular level upon the study of permeability, aging indoors and outdoors of polymers, the role of low MW impurities. Many polymers or polymeric coatings prepared by free-radical photopolymerization (UV-cure) have residual photo initiators (PI). Presence of PI and peculiarities of a chemical structure of coatings affect their such important property as weatherability.

Relatively hard polymer may demonstrate a low viscous drug for translational and rotational diffusion of low MW species possibly due to a high free volume $V_{\mathrm{f}}$ and existence of channels for diffusion. At the same time soft polymer can manifest a hindered diffusion, say due to multiple entanglements in its structure. Analysis of ESR spectra of stable free radicals (usually nitroxyls) inside polymers gives valuable info on polymer structure, mobility of polymer chains, $V_{f}$ etc. This method of spin labels and spin probes has been used for many decades. This method mainly addresses rotational diffusion of stable radicals [1-5].

Another useful method exploits mainly translational diffusion of reactive free radicals. Reactive free radicals generated in a polymer one or another way, undergo self-termination:

$$
\mathrm{R}_{1}+\mathrm{R}_{2} \rightarrow \text { Diamagnetic product }(\mathrm{s})
$$

$$
\mathrm{R}_{1}+\mathrm{R}_{1} \rightarrow \text { Diamagnetic product(s) }
$$

Self-termination can be either recombination of radicals or their disproportionation. Usually both processes are called recombination when products are of modest interest. Radicals which meet each other in the course of random diffusion wandering in polymer form so-called free or F-pairs under an encounter. Radicals recombination occurs within F-pairs. Rates of reaction (1) for highly reactive radicals depend upon the viscosity of a polymer matrix.

Radicals are usually generated as pairs. For example, photodegradation of a polymer often occurs by a cleavage of a weak chemical bond upon absorption of photon. Such pair of radicals which exists for some short time is called geminate or G-pair:

$$
\begin{gathered}
\mathrm{hv} \\
\mathrm{R}_{1} \mathrm{R}_{2} \rightarrow\left[\mathrm{R}_{1}, \mathrm{R}_{2}\right] \\
\text { Polymer chain G-pair }
\end{gathered}
$$

It is convenient in the lab experiments to photogenerate G-pair exciting by short light flashes one or another precursor of an RP. Benzophenone (BP) is a common PI of photopolymerization. The cured polymer (coating) has a residual BP. BP becomes a dangerous additive, which initiate photodegradation of a prepared polymer via formation of G-pairs:

$$
{ }^{3} \mathrm{BP}+\mathrm{R}_{1} \mathrm{R}_{2} \rightarrow{ }^{3}\left[\mathrm{BPH}^{\bullet}, \mathrm{R}_{1} \mathrm{R}_{2}(-\mathrm{H})^{\bullet}\right]
$$

BP being in the excited triplet state (superscript 3 in eq.3) abstracts hydrogen from a polymer chain. The spin state is preserved during a chemical reaction, and the formed RP is in a triplet state as well, see eq. 3 . 
At the same time, BP turns out to be a very good probe to study photo resistance of a polymer and its molecular properties. Corresponding free radical $\mathrm{BPH}^{\bullet}$ of $\mathrm{BP}$ has the known absorption spectrum in the visible area of the spectrum; its disappearance can be easily monitored by transient absorption spectroscopy.

The notion of G-pair is like that of solvent (polymer) cage. Radicals generated in cages either escape the cage or recombine within a cage:

This competition of two reactions is obviously sensitive to the properties of the cage, i.e., to the properties of polymer. There is a way to generate a couple of the same radicals as F- or as G-pair and get additional information on the media comparing reactivity of radicals in two types of pairs.

External magnetic field affects reactivity of radicals in Fand especially in G-pairs. Magnetic field effects depend also on properties of a polymer matrix. Experimental observations in the sprit described above allowed to make valuable for material science conclusions.

\section{Acknowledgement}

None.

\section{Conflict of Interest}

No conflict of interest.

\section{References}

1. PP Levin, AE Efremkin, IV Khudyakov (2017) Benzophenone as a photoprobe of polymer films. Chem Phys 495: 23-28.

2. PP Levin, AF Efremkin, AV Krivandin, SM Lomakin, OV Shatalova, et al. (2018) Photoinduced Reactions of Benzophenone in Biaxially Oriented Polypropylene. J Phys Chem A 122(17): 4298-4305.

3. Khudyakov IV, Turro NJ (2010) In Carbon-Centered Free Radicals and Radical Cations. Structure, Reactivity, and Dynamics; Forbes MDE (Ed) Wiley: Hoboken, NJ, USA, pp. 249-279.

4. IV Khudyakov, NJ Turro (2010) Cage Effect Dynamics under Photolysis of Photoinitiators. Des Monom Polym 13(5): 487-496.

5. IV Khudyakov, RD Zopf, NJ Turro (2009) Polyurethane Nanocomposites. Des Monom Polym 12: 279-290. 